\title{
Quantum Correlations in Two-Particle Anderson Localization
}

\author{
Yoav Lahini ${ }^{1}$, Yaron Bromberg ${ }^{1}$, Demetrios N. Christodoulides ${ }^{2}$, and Yaron Silberberg ${ }^{1}$ \\ ${ }^{1}$ Department of Physics of Complex Systems, Weizmann Institute of Science, Rehovot, Israel. and \\ ${ }^{2}$ CREOL/College of Optics, University of Central Florida, Orlando, Florida, USA
}

\begin{abstract}
We predict the quantum correlations between non-interacting particles evolving simultaneously in a disordered medium. While the particle density follows the single-particle dynamics and exhibits Anderson localization, the two-particle correlation develops unique features that depend on the quantum statistics of the particles and their initial separation. On short time scales, the localization of one particle becomes dependent on whether the other particle is localized or not. On long time scales, the localized particles show oscillatory correlations within the localization length. These effects can be observed in Anderson localization of non-classical light and ultra-cold atoms.
\end{abstract}

More than fifty years ago, P.W. Anderson predicted that a single quantum particle released in a disordered lattice can exhibit exponential localization in space [1], a phenomenon termed Anderson Localization (AL). Since then there has been an ongoing effort to observe the signature of AL experimentally, for example using light [2]. Recently a novel approach enabled the direct observation in space of $\mathrm{AL}$ for photons [3] [6] and ultra-cold atoms [7]. These experiments, reporting the exponential localization of the particle density distribution, agree with the predictions of the single particle model as long as no interactions are involved.

The question arises, whether there are measurable phenomena that are not described by the single particle model when considering the localization of a few photons or atoms. It is known that when indistinguishable particles propagate together, exchange terms can result in the formation of correlations between their positions even in the absence of interactions. This result, known as the Hanbury Brown-Twiss (HBT) effect [8], was studied theoretically and experimentally for particles propagating in free space. It was observed that quantum statistics play a crucial role in the formation of HBT correlations - bosons tend to bunch, while fermions exhibit antibunching [8, 9]. Yet, it is not clear how HBT correlations evolve when the particles propagate through disordered media and become localized. While correlations were recently observed in the multiple-scattering of non-classical light [10 12], experiments on AL focused on the particle density distribution, and therefore could not observe spatial correlations between the localized particles. Now, experimental techniques that allow a direct observation of localization [5-7] can provide access to the study of HBT correlations between particles exhibiting AL.

In this Letter we predict the correlations between two indistinguishable quantum particles evolving simultaneously in disordered lattices. We consider non-interacting particles, and therefore the particle-density follows the single particle dynamics: both particles undergo Anderson localization. Nevertheless, we find that the two particles develop non-trivial spatial correlations due to interferences of all the scattering paths the two particles
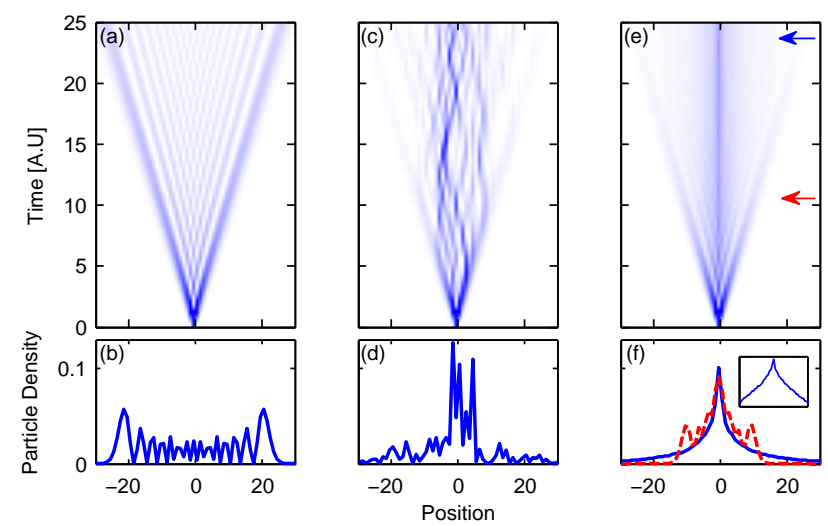

Figure 1: (color online). Dynamics of a single quantum particle placed at $t=0$ on a single site. (a) In a periodic lattice, the particle density distribution expands ballistically, with high density at the edges of the distribution. (b) Cross-section of the density distribution after some evolution. (c),(d) In a disordered lattice, the expansion is limited to a finite region. (e) The density distribution, averaged over 1000 realizations of disorder. The expansion starts ballistically. After some propagation a localized component emerges around the initial position, (red arrow, red cross-section in (f)). The ballistic component decays in time, leaving the distribution exponentially localized (blue arrow, blue cross-section in (f)).

can take as a pair. On short time scales, the localization of one of the particles uniquely determines whether the other particle will be localized or not. On longer time scales, when both particles localize, the particles exhibit oscillatory correlations within the localization length. Remarkably, these oscillatory correlations survive multiple scattering even after very long evolution times. In addition, we show that fermionic correlations can be reproduced by entangled bosonic states.

To study two-particle localization, we follow the formalism developed in [13] for periodic systems. We consider a one dimensional quantum tight-binding model, given by Hamiltonian:

$$
H=\sum_{n} W_{n} a_{n}^{\dagger} a_{n}-\sum_{<n, m>} T_{n, m} a_{n}^{\dagger} a_{m}
$$


where $a_{n}^{\dagger}$ is the creation operator for a particle in site $n$, $W_{n}$ is the on-site energy and $T_{n, m}$ is the tunneling amplitude between nearest neighbors. At time $t$ the creation operator at site $r$ is given by

$$
a_{r}^{\dagger}(t)=\sum_{r^{\prime}} U_{r r^{\prime}}(t) a_{r^{\prime}}^{\dagger}(t=0)
$$

where the time-evolution operator $U_{r r^{\prime}}(t)=\left(e^{i \hat{H} t}\right)_{r, r^{\prime}}$ is a unitary transformation given by calculating the exponent of the Hamiltonian matrix $\hat{H}$, and describes the amplitude for transition of a single particle located at site $r^{\prime}$ at $t=0$, to site $r$ at time $t$. In the following, we study the evolution of two indistinguishable particles in a disordered lattice, where disorder is introduced by randomizing the tunneling amplitudes. We focus on the particledensity $n_{r}(t)=\left\langle a_{r}^{\dagger} a_{r}\right\rangle$ and on the two-particle correlation $\Gamma_{q, r}(t)=\left\langle a_{q}^{\dagger} a_{r}^{\dagger} a_{r} a_{q}\right\rangle$, highlighting the single-particle versus two-particle features of the dynamics.

We start by considering the evolution of a single particle, described by the particle density $n_{r}(t)=\left|U_{r r^{\prime}}(t)\right|^{2}$, where $r^{\prime}$ is the location of the particle at $t=0$. In Fig. 1 we plot the evolution of the particle density for an initial condition $a_{0}^{\dagger}|0\rangle$, i.e. a single particle at site number 0 . Fig. 1a depicts the propagation of the particle in a periodic lattice, which is given by $n_{r}=J_{r-r^{\prime}}^{2}(2 T t)$, where $J_{n}$ is the Bessel function of order $n$. This dynamics is known as a quantum walk [13 16], in which the particle density expands ballistically (i.e. linearly in time), and the probability to find the particle is highest at the edges of the distribution (see Fig 1b). When disorder is introduced (here by randomizing the tunneling amplitudes), the propagation changes significantly, as shown in Fig. 1c,d. A statistical average of the probability distribution over many realizations of disorder (Fig 1e,f) reveals a clear cross-over from ballistic expansion at short times to exponential localization at long times (blue arrow in Fig 1e). At intermediate times (red arrow in Fig. 1e), the particle can be either localized or ballistic [6].

We now consider two non-interacting particles which are initially localized on sites $r^{\prime}$ and $q^{\prime}$, focusing on the correlations between their locations at a later time $t$. The correlation matrix is given by $\Gamma_{q, r}=\left\langle\langle| U_{q r^{\prime}} U_{r q^{\prime}} \pm\right.$ $\left.\left.\left.U_{q q^{\prime}} U_{r r^{\prime}}\right|^{2}\right\rangle\right\rangle[13]$. Here $\langle\langle\cdot\rangle\rangle$ signifies averaging over realizations of disorder, and the upper and lower signs describe, respectively, bosons and fermions of same spin. The depicted matrices (for example in Fig. 2) represent the probability to detect at time $t$ exactly one particle at site $q$ and one particle at site $r$. As we show below, the density distribution is identical for bosons and for fermions (see Fig. 2e), yet the emerging quantum correlations between the particle pair depends on the initial position of each particle and on their quantum statistics.

We first consider the case in which initially the two particles are positioned at two adjacent sites in a separable state, i.e. the initial condition is given by $|\phi\rangle=a_{1}^{\dagger} a_{0}^{\dagger}|0\rangle$.
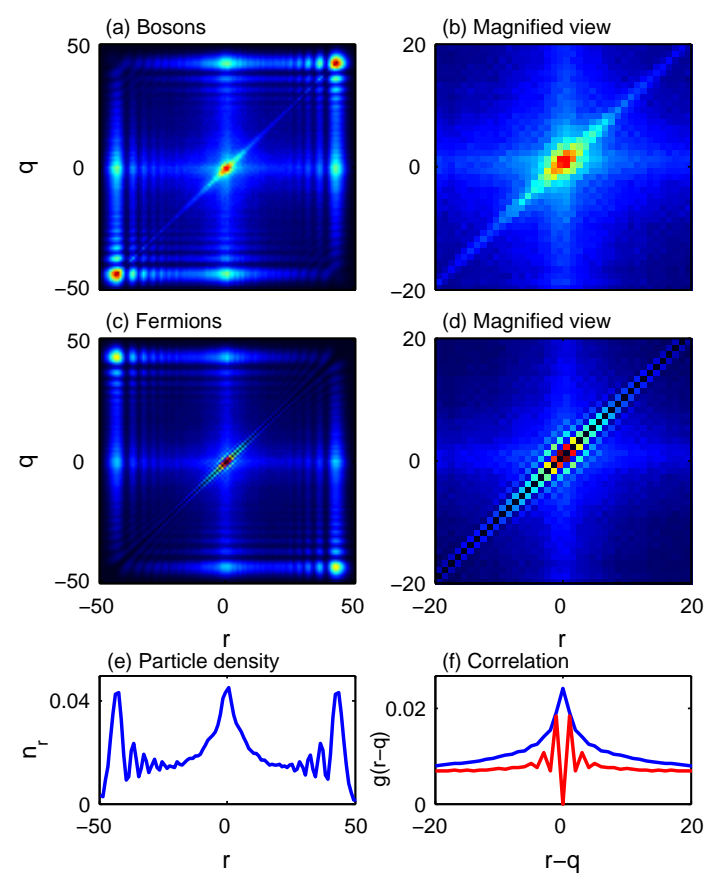

Figure 2: (color online). Two-particle correlation function in disordered lattices. At $t=0$, two particles are placed at two neighboring sites. The correlation function is calculated after a short evolution, where remnants of the ballistic component are still present (see Fig. 1e,f), averaged over a 1000 disorder realizations. The matrices represent the probability to detect one particle at site $q$ and one particle at site $r$. (a) Bosons either scatter and become localized, or remain ballistic. However, if they both remain ballistic, they always reach the same side of the distribution. (b) Magnification of the localized component at the center of the correlation map. (c) Fermions show similar behavior, only that if they both remain ballistic they always separate to different sides of the distribution. (d) Magnification of the correlation map, showing a checker-like pattern. (e) The particle density distribution, which is identical for fermions and for bosons, computed by tracing out the position of one of the particles (summing over the columns of the correlation matrix). (f) The inter-particle distance probability for bosons (blue) and fermions (red). Bosons tend to appear at the same site, while fermions are more likely to be separated by an odd number of sites.

We calculate the two-particle correlation function after a relatively short evolution time, in which each particle has a non-zero probability to be localized or to remain ballistic (see Fig. 1). As shown by the correlation matrix in Fig. 2a, if the two particles are bosons they can both remain ballistic (corners of the correlation matrix), or both become localized (center of the correlation matrix). The possibility for one boson to localize and the other to remain ballistic is also non-zero. However, when both bosons exhibit ballistic behavior, the probability for the two bosons to separate to two different sides of the distribution vanishes (top left and bottom right corners of the correlation matrix). This is the signature of bosonic bunching in lattices [13]. In this case then, the 

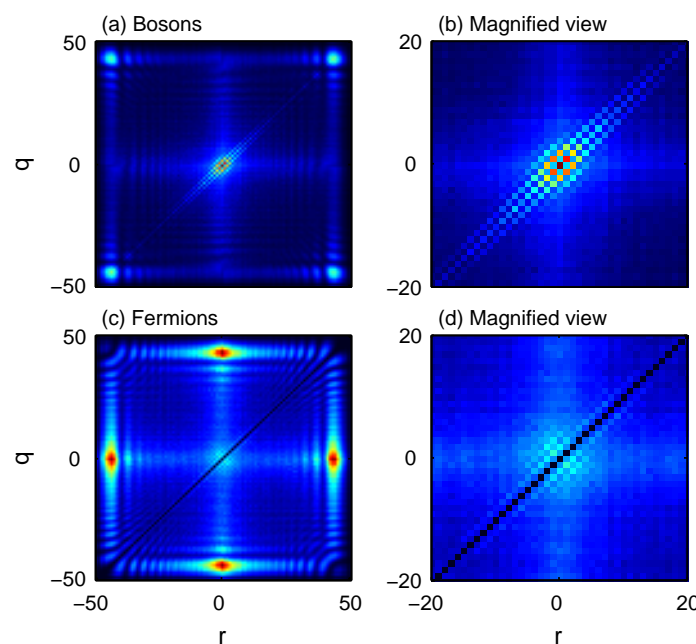

(d) Magnified view
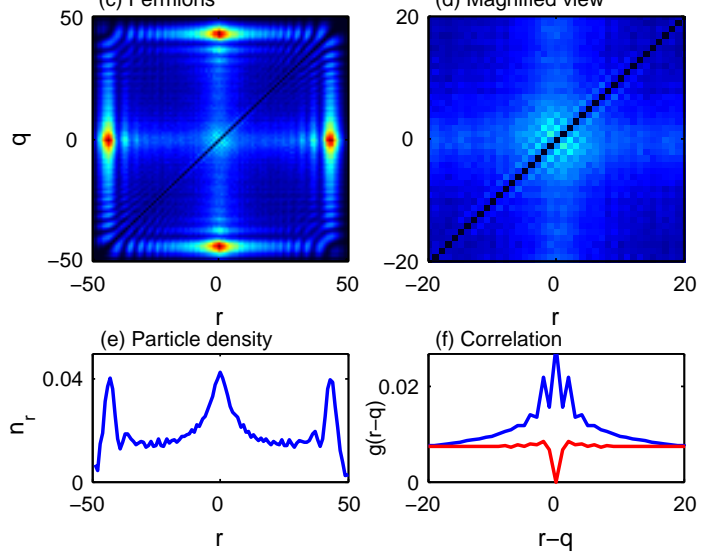

Figure 3: (color online). The two-particle correlation function when At $t=0$, the two particles are placed at two nonadjacent sites. (a) Bosons show two possible effects - they either both localized, or both remain ballistic. (b) Closeup on the correlation inside the localized component. (c) Fermions almost always split - one fermion becomes localized and the other remains ballistic. (d) Closeup on the correlation inside the localized component. (e) The particle density distribution, identical for fermions and bosons. (f) The inter-particle distance probability within the localized regime for bosons (blue line) and fermions (red line). The fermions exhibit a flat inter-particle distance distribution (except at zero separation), while the bosons show an oscillating probability.

two-particle correlation has two components - a ballistic component showing spatial bunching, and a localized component without spatial correlations. A closeup on the correlation in the localized component is given in Fig. $2 \mathrm{~b}$, also describing the correlation matrix at later times, when the distribution is completely localized [17].

In the case of fermions, the correlation matrix of Fig. $2 k$, d shows that if the two particles remain ballistic (i.e. both particles did not localize yet), they will exhibit antibunching and separate to opposite sides of the distribution. A closeup of the correlations inside the localized component reveals a checkers-like pattern, meaning that even when both fermions are localized they are non-trivially arranged in space. Most significantly, this checker-like pattern survives the random scattering process even after very long evolution times [17].

To understand the significance of the checker-like pattern we extracted from the correlation matrix the interparticle distance probability given by $g(\Delta)=\sum_{q} \Gamma_{q, q+\Delta}$. Comparison between the results for bosons and fermions are depicted in Fig. 2:. Bosons (blue line) have the high- est probability to be at the same site, and the probability drops monotonously with distance. In contrast, fermions have zero probability to be on the same site (as expected due to Pauli exclusion). Yet, interestingly, the correlation oscillates: the two fermions tend to be separated by an odd number of sites.

An even more dramatic effect takes place when the initial condition is such that the two particles are placed at non-adjacent sites. Consider the initial condition $|\phi\rangle=a_{-1}^{\dagger} a_{1}^{\dagger}|0\rangle$ - two particles at two sites separated by an empty site. Again we calculate the correlation function after a relatively short propagation, in which the dynamics includes both a ballistic and a localized component. For bosons (Fig. 3a) there are only two possibilities: either the particles are both localized (center of the correlation matrix) $O R$ they both remain freely propagating (corners of the correlation matrix). The possibility for one particle to be localized and the other one to be free is diminished to nearly zero. A closeup on the correlation when both particles are localized (Fig. 3b) shows that in this case it is the bosons that exhibit the checkers-like pattern; they are more likely to be separated by an even number of sites.

For this input state, fermions exhibit a different symmetry in the correlations (Fig. 3r): the two particles tend to always occupy different components of the expanding wavepacket: one fermion becomes localized, while the other fermion remains freely propagating in the ballistic component. The closeup on the localized regime (Fig. 3. d) shows a flat distribution, except for the zero probability to be at the same site. The inter-particle distance for this case is depicted in (Fig. 3f) [17].

Finally, it is of interest to consider the propagation in disordered lattices of path-entangled input bosonic states, which can be regularly generated in quantumoptics experiments. In a lattice system, such a state is given by a superposition of two bosons - placed together at one site $O R$ another site $|\phi\rangle=\frac{1}{2}\left[\left(a_{r^{\prime}}^{\dagger}\right)^{2}+e^{i \theta}\left(a_{q^{\prime}}^{\dagger}\right)^{2}\right]|0\rangle$. Interestingly, properly constructed entangled states reproduce some of the features presented by fermions. In Fig 4 we present the calculated correlation matrices for two kinds of path-entangled input states: In Fig. 4a, we plot the correlation matrix when the initial state is $|\phi\rangle=\frac{1}{2}\left[\left(a_{0}^{\dagger}\right)^{2}+\left(a_{1}^{\dagger}\right)^{2}\right]|0\rangle$. The correlation matrix shows several features that are similar to the ones exhibited by the fermions as seen in Fig. 2 $2 c$ : the two particles can both become localized, both remain ballistic, or split - one localized and one ballistic. However, they never end up on the same ballistic lobe. The main difference is in the localized region: the checker pattern disappears, and the diagonal $q=r$ of the correlation matrix now shows enhanced probability. In Fig. 45 we plot the correlation matrix when the initial state is $|\phi\rangle=\frac{1}{2}\left[\left(a_{-1}^{\dagger}\right)^{2}+e^{i \pi}\left(a_{1}^{\dagger}\right)^{2}\right]|0\rangle$. i.e. the two states are separated by an empty site. This time the pair shows a tendency to split - one boson is localized, while the 

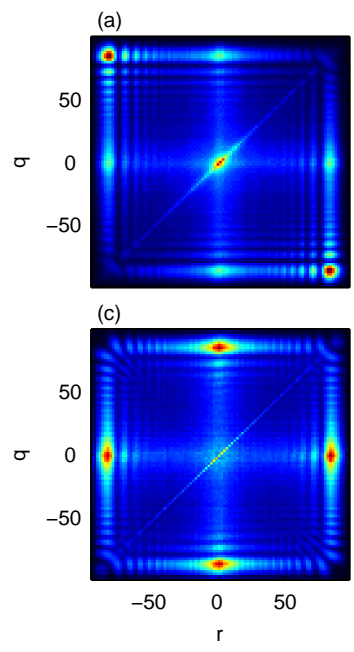
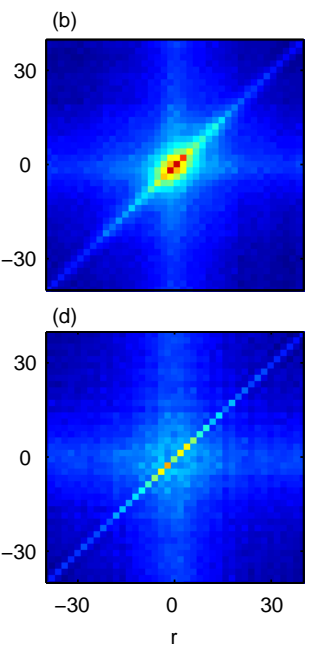

Figure 4: (color online) Correlation maps for path-entangled bosons evolving in disordered lattices. (a) Correlation map for the initial condition $|\phi\rangle=\frac{1}{2}\left[\left(a_{0}^{\dagger}\right)^{2}+\left(a_{1}^{\dagger}\right)^{2}\right]|0\rangle$. (b) Magnified view of the localized component. (c) For $\phi=\frac{1}{2}\left[\left(a_{-1}^{\dagger}\right)^{2}-\right.$ $\left.\left(a_{1}^{\dagger}\right)^{2}\right]|0\rangle$. (d) Magnified view. These correlations are similar to the fermionic results in Fig. 2c,d and 3c,d (see text).

other remains ballistic. The correlation matrix is similar to that exhibited by the fermion pair in Fig. 35, except the enhanced probability on the matrix diagonal $q=r$.

The emergence of the checker-like patterns in the correlations can be explained in terms of the lattice eigenmodes excited in each case. Disordered lattices support two kinds of eigenmodes: flat-phased and staggered, in which adjacent sites are in phase $/ \pi$ out of phase, correspondingly [6]. Certain initial conditions, (depending on the initial distance between the two particles and their quantum statistics) involve the simultaneous excitations of both kinds of eigenmodes, resulting in a density pattern that contains a component with two-site periodicity. This effect is washed out in the density distribution averaged over all realizations of disorder, as in each realization the oscillations appear in a different location. However, the fact that such oscillations appears in each realization will be recorded in the averaged correlation.

At higher dimensional disordered systems, the single particle dynamics can be qualitatively different. For example expanding wavepacket can exhibit diffusive expansion before localization [5]. Nevertheless, we have verified that the results described above appear also in two dimensional lattices, including the checker-like patterns.

In conclusion, we studied the evolution of two noninteracting quantum particles in disordered lattices exhibiting Anderson localization. The two-particle correlation exhibits unique features that survive the random scattering, such as oscillating correlations and correlated cross-over from ballistic propagation to localization. The correlations depend on the initial separation between the particles and on their quantum statistics. Experimentally, the bosonic correlations can be observed by injecting photon pairs generated using spontaneous parametric down conversion, into disordered waveguide lattices. Such lattices were recently used to observe Anderson localization of classical light [5, 6], and were shown to be feasible for observing non-classical correlations between photon pairs [13, 15, 18]. With atom-matter waves, correlations can be observed in the quantum walks [16] of a pair of atoms in disordered lattices, offering a possibility to study the effect of interaction [20] on correlations. Alternatively, Density-density correlations can be measured for expanding Bose-Einstein condensates in disordered optical potentials [7, 19].

This work was supported by the German - Israel Foundation (GIF), the Minerva Foundation and the Crown Photonics Center.

[1] P. W. Anderson, Phys. Rev. 109, 1492 (1958).

[2] M. Storzer et. al., Phys. Rev. Lett. 96, 063904 (2006); D. S. Wiersma et. al., Nature 390, 671 (1997) ;M. P. Van Albada and A. Lagendijk, Phys. Rev. Lett. 55, 2692 (1985); P. E. Wolf and G. Maret, Phys. Rev. Lett. 55, 2696 (1985); A. A. Chabanov, M. Stoytchev, and A. Z. Genack, Nature 404, 850 (2000).

[3] H. De Raedt, A. Lagendijk, and P. de Vries, Phys. Rev. Lett. 62, 47 (1989).

[4] T. Pertsch et. al., Phys. Rev. Lett. 93, 053901 (2004).

[5] T. Schwartz et. al., Nature 446, 52 (2007).

[6] Y. Lahini, et. al., Phys. Rev. Lett. 100, 013906 (2008).

[7] J. Billy, et. al., Nature 453, 891 (2008); G. Roati et. al., Nature 453, 895 (2008); J. Chabe et. al., Phys. Rev. Lett. 101, 255702 (2008).

[8] R. Hanbury Brown and R.Q. Twiss, Nature 177,27 (1956).

[9] T. Jeltes et al., Nature 445, 402 (2007); T. Rom et al., Nature 444, 733 (2006); S. Folling et al., Nature 434, 481 (2005); M. Schellekens et al., Science 310, 648 (2005); M. Henny et al., Science 284, 296 (1999); I. Neder et al., Nature 448, 333 (2007); H. Kiesel et al., Nature 418, 392 (2002).

[10] C. W. J. Beenakker, J. W. F. Venderbos, and M. P. van Exter, Phys. Rev. Lett. 102, 193601(2009).

[11] S. Smolka et al., Phys. Rev. Lett. 102, 193901(2009).

[12] W. H. Peeters, J. J. D. Moerman, and M. P. van Exter, Phys. Rev. Lett. 104, 173601 (2010).

[13] Y. Bromberg et al., Phys. Rev. Lett. 102, 253904 (2009).

[14] J. Kempe, Contemp. Phys., 44, 307, (2003).

[15] H. B. Perets, et al, Phys. Rev. Lett. 100, 170506 (2008).

[16] M. Karski, et al, Science 325, 174 (2009).

[17] See EPAPS Document No. [] for animation. For more information on EPAPS, see http://www.aip.org/pubservs/epaps.html

[18] A. Politi et al., Science 320, 646-649 (2008); A. Rai, G.S. Agarwal and J. H. H. Perk, Phys. Rev. A 78, 042304 (2008); R. Keil et. al., Phys. Rev. A 81, 023834 (2010); A. Peruzzo et al., arXiv:1006.4764 (2010).

[19] E. Altman, E. Demler, and M. D. Lukin, Phys. Rev. A 
70, 013603 (2004).

[20] D. L. Shepelyansky, Phys. Rev. Lett. 73, 2607 (1994). 